\title{
Zum 75. Geburtstag von Ulrich Lanz
}

\section{Personal Congratulations on occasion of the $75^{\text {th }}$ Birthday of Ulrich Lanz}

R. E. Giunta, H. Krimmer, $M$. Sauerbier,

K.J. Prommersberger
Bibliografie

DOI http://dx.doi.org/

10.1055/s-0041-110029

Handchir Mikrochir Plast Chir

2015; 47: 410-411

(c) Georg Thieme Verlag KG

Stuttgart · New York

ISSN 0722-1819

Korrespondenzadresse

Univ.-Prof. Dr. med.

Riccardo E. Giunta

Handchirurgie, Plastische

Chirurgie und Ästhetische

Chirurgie

Klinikum der Ludwig-

Maximilians

Universität (LMU) München

Pettenkoferstraße 8a

80336 München

r.giunta@med.uni-muenchen.de
Liebe Leserinnen und Leser,

zu Ehren des 75. Geburtstag unseres langjährigen Herausgebers der Zeitschrift Handchirurgie, Mikrochirurgie, Plastische Chirurgie im November 2015 möchten wir folgende Eindrücke von Schülern, die von Ulrich Lanz geprägt wurden, für Sie zusammen fassen, uns bei ihm bedanken und natürlich ganz besonders herzlich gratulieren:

Es ist 20 Jahre her, dass ich 1995 und 1996 bei Ulrich Lanz in Bad Neustadt an der Saale als Assistenzarzt tätig war. Zu dieser Zeit war Ulrich Lanz gerade wenige Jahre von der Universitätsklinik Würzburg an das privat geführte RhönKlinikum gewechselt und ist damit einen Weg gegangen, den viele Repräsentanten kleinerer Fachgebiete, die an Universitätskliniken nicht oder nur ungenügend Gelegenheit zur Weiterentwicklung und Gestaltung Ihres Fachgebiets finden, gehen müssen. Für Ulrich Lanz war dies die logische und richtige Entscheidung. In Bad Neustadt hat er die Unterstützung vorgefunden, die ihm ermöglichte, ein Team aufzubauen, welchem er die Faszination für die Handchirurgie auf einem akademischen Niveau vermitteln konnte. $\mathrm{Er}$ hat dort die größte und bedeutendste Handchirurgie in Deutschland dieser Zeit aufgebaut. Viele Kollegen, die zu jener Zeit in Bad Neustadt tätig waren, sind Dank seiner Inspiration und Anleitung zu wichtigen akademischen Vertretern der Handchirurgie über die Jahre gereift und haben wichtige Positionen in den Fachgesellschaften übernommen.

„The Seed has born Fruit“(„Der Samen ist aufgegangen“) war der Titel von Ulrich Lanz diesjährigem Vortrag zu Anlass des 70. amerikanischen Handchirurgen Kongress der ASSH in den USA. Er hat die Faszination, die er selbst bei Robert E. Caroll in New York erfahren hat, in Deutschland „aufgehen“ lassen und unter uns in den nachfolgenden Generationen weiter verbreitet. Was kann man sich als akademischer Chirurg mehr wünschen?

Ich selbst habe Ulrich Lanz als einen der sehr wenigen herausragenden Persönlichkeiten in meinem bisherigen Berufsleben erlebt. Nicht wegen seiner Position als Chef - wie leider bei den meisten Führungskräften - hat er Autorität ausgestrahlt und wurde ihm Respekt entgegen gebracht. Vielmehr hat er wegen seiner Kompetenz, seines Committments und seiner Begeisterung für das Fachgebiet im besten Sinne „Leadership“ ausgestrahlt. In diesem Sinne ist und wird er mir immer ein großes Vorbild für meine eigene

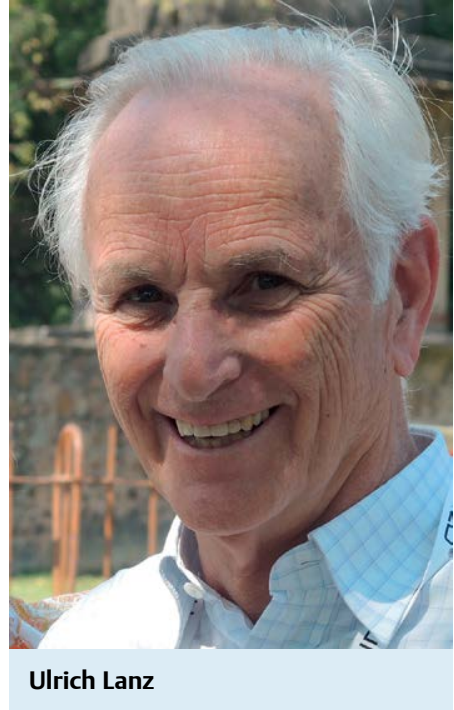

weitere berufliche Entwicklung sein. Ich schätze mich außerordentlich glücklich, dass wir im Laufe der Jahre eine Freundschaft über die Generationen aufgebaut haben und auch viele Interessen im beruflichen wie privaten Bereich teilen.

Lieber Ulrich, ganz herzlichen Glückwunsch zu Deinem 75. Geburtstag!

Riccardo Giunta

München im November 2015

Lieber Ulrich,

Du hast den beruflichen Werdegang vieler Kollegen durch Deine Begeisterung für das Fach Handchirurgie maßgeblich geprägt. Nach dem Aufbau der Handchirurgie an der Universität Würzburg mit überregionaler Reputation ist es Dir dann gelungen mit der Klink für Handchirurgie in Bad Neustadt ein weltweit anerkanntes Zentrum zu etablieren. Mein Glück war es, Dich dabei begleiten zu dürfen und hinsichtlich operativer und wissenschaftlicher Tätigkeit von Dir zu lernen. Immer hast Du uns dezent stimuliert neben der operativen Tätigkeit, uns auch wissenschaftlich $\mathrm{zu}$ engagieren und damit die Voraussetzungen für beruflichen Erfolg zu schaffen. Besonders hervorzuheben, ist der internationale Spirit, den Du uns frühzeitig vermittelt hast, und immer wenn wir im Ausland unterwegs waren und der Name Lanz fiel, waren wir geschätzt und willkommen. Gerne erinnere ich mich an gemeinsame Kongressbesuche, die für uns dank Deiner Leidenschaft für das Fliegen mit Dir als Piloten ein ganz besonderes Erlebnis darstellten. Unsere sportlichen Aktivitäten, insbesondere das ge- 
meinsame jährliche Skifahren fanden von und mit Dir volle Unterstützung. Langjähriger Teamgeist, der bis heute zu nachhaltigen Freundschaften mit vielen Kollegen geführt hat, ist Dir und Deiner persönlichen Art zu verdanken. Alles Gute zu Deinem 75jährigen Jubiläum und vielen Dank für Alles, was Du für uns und die Handchirurgie getan hast.

\section{Dein Hermann Krimmer}

Ravensburg im November 2015

\section{$\nabla$}

Als Medizinstudent an der Bayerischen Julius-MaximiliansUniversität zu Würzburg hatte ich 1989 das große Glück Ulrich Lanz als damaligen Leiter der Handchirurgie an der Chirurgischen Universitätsklinik kennenzulernen. Er war national und international höchst anerkannt und seine Reputation über alle Grenzen hinaus außergewöhnlich. Seine Vorlesungen über die Handchirurgie faszinierten mich sofort, und ich wollte unbedingt von ihm lernen. Nach einer ersten Famulatur in seinem Bereich, hatte ich die Gelegenheit unter seiner Leitung und der Betreuung durch Hermann Krimmer eine Dissertation zu beginnen (Fortgeschrittener Karpaler Kollaps [SNAC-Wrist] bei Skaphoidpseudarthrose; Therapiekonzept: Mediokarpale Teilarthrodese).

Durch die exzellente internationale Vernetzung von Ulrich Lanz und seine Empfehlung konnte ich Teile meines Praktischen Jahres im Universitätsspital Zürich bei Viktor E. Meyer sowie am Massachussetts General Hospital in Boston bei Richard H. Gelberman und Jesse B. Jupiter absolvieren. Nach dem 3. Staatsexamen im November 1993 hatte ich das Glück eine Stelle in der neu gegründeten Klinik für Handchirurgie in Bad Neustadt an der Saale antreten zu dürfen und bis 1995 dort zunächst als Arzt im Praktikum und später als Assistenzarzt zu arbeiten. Erwartungsgemäß entwickelte sich diese Klinik unter seiner Leitung als weltweit anerkannte Institution. Die Zeit dort war für mich sehr spannend und lehrreich, und ich empfand es als großes Privileg von Ulrich Lanz so viel über das wunderbare Fach der Handchirurgie zu erfahren und seine chirurgische Brillianz gepaart mit außergewöhnlichem Verständnis für die Anatomie im Operationssaal beobachten zu können. 1995 wurde ich dann von Ulrich Lanz promoviert, und die Handchirurgie entwickelte sich erfreulicherweise zu einem wesentlichen Teil meines weiteren beruflichen Lebens.

Ulrich Lanz ist für mich immer schon ein großes Vorbild, und ich konnte ihn nicht nur als meinen ersten chirurgischen und akademischen Lehrer erleben, sondern auch als einen Arzt, der für seine Patienten immer da war und das bestmögliche, aber auch das vernünftigste wollte. Seine Entscheidungen für den einen oder den anderen Eingriff waren immer von Augenmaß und Achtung der Persönlichkeit der Patienten geprägt. Noch heute denke ich manchmal bei schwierigen Pathologien darüber nach, wie er sich wohl entscheiden würde.

Alle diese Qualitäten, aber auch die Art und Weise wie er die Klinik in Bad Neustadt führte und mit „Teamgeist“ erfüllte, würden heute neudeutsch wohl „Excellence in Leadership“ genannt. Ich bin daher in höchstem Maße dankbar, dass ich einer der Schüler von Ulrich Lanz sein durfte.

Lieber Ulrich Lanz, alles Gute zum 75. Geburtstag verbunden mit Gesundheit und Lebensfreude!

Michael Sauerbier

Frankfurt im November 2015
Als ich im September 1994 an die Klinik für Handchirurgie Bad Neustadt kam, wollte ich eigentlich nur 3 Jahre bleiben, um dann als Handchirurg zurück in die Unfallchirurgie zu kehren. Dass ich mit einer $1 \frac{1}{2}$ jährigen Unterbrechung nun bereits über 20 Jahre in Bad Neustadt bin, dafür gibt es einen Grund: Ulrich Lanz.

Ulrich Lanz war und ist mir Lehrer und Vorbild zugleich. Er war ein akribischer Operateur, ein Perfektionist und gerade deshalb vielleicht maximal selbstkritisch. Op-Assistenzen bei ihm waren Lehrstunden in Theorie und Praxis. Sein Operieren war ästhetisch, ohne dass er es jedoch zelebrierte. Seine Therapieentscheidungen in der Sprechstunde basierten auf einer minutiösen klinischen Untersuchung, einer maximal strukturierten Analyse der Bildgebung und nicht zuletzt auf der großen Geduld, mit der er Patienten zuhören konnte. Und, er war bereit sich der Probleme der Patienten anzunehmen.

Hier einige meiner Erlebnisse mit ihm und einige Zitate von ihm. Vielleicht können sie besser vermitteln, was ich nur unzureichend beschreiben kann.

Als ich ihn einmal in der Op-Umkleide nach einer schwierigen Operation traf: „Ich weiß nicht, ob ich dem Patienten was Gutes getan habe“.

Eine Operation endete für ihn nicht mit der Hautnaht, sondern mit dem Anlegen des (Gips-) Verbandes. Als er bei einem der Skiwochenenden der Abteilung kurz vor der Abfahrt des Buses sah, dass ein von einem von uns angelegter Gipsverband nicht korrekt war, blieb er zurück, legte einen neuen Gipsverband an und kam mit seinem Privat-PKW nachgefahren. - „Um den Verband nach einer Dupuytren-Operation korrekt anzulegen, muss man mindestens 3 Jahre Handchirurgie gemacht haben“.

Die wissenschaftliche Aufarbeitung des eigenen Patientengutes war für ihn eine conditio sine qua non für eine erfolgreiche klinische Arbeit. „Nur operieren macht dumm“. „Der wichtigste Schritt in meiner Karriere war der Kauf meiner Fotokamera“.

Auch wenn er Schreibfehler nicht mit roter Tinte ausbesserte, so konnte er sich doch einen Kommentar zu dem einen oder anderen Schreibfehler und der nichtkorrekten Aussprache eines Eigennamens nicht verkneifen. „Man kann einen Finger mit einem großen „D“ gefolgt von einer arabischen Ziffer abkürzen, also z.B. „D3“ für den Mittelfinger oder mit einer römischen Ziffer (III). Wer aber „DIII“ schreibt, zeigt, dass er nichts von Handchirurgie versteht“. „Bunnell schreibt man mit 2 „n“ und 2 „1“ und spricht es, wie man's schreibt und nicht „Bannel“.“ „Lichtman spricht sich „Liktmen“.

Wenn es auch grundsätzlich sein Bestreben war, anatomische Verhältnisse und Funktion so gut wie irgend möglich wieder herzustellen, verlor er doch nie den jeweiligen Patienten und dessen Bedürfnisse aus dem Auge und entschied sich dort, wo er keinen Gewinn für den Patienten sah, auch gegen eine mögliche Operation. „Junge Soldaten haben Patronen in ihrer Tasche, alte Schokolade“.

Zuletzt der wichtigste Satz zu mir (3.1.1995): „Herr Prommersberger, ab heute kümmern Sie sich um den Radius“.

Noch heute frage ich Ulrich Lanz immer wieder um seinen Rat, schwierige Fälle betreffend, problematische Manuskripte und auch persönliche Dinge. Ich hoffe sehr, dass ich das noch lange kann, und wünsche ihm zu seinem 75. Geburtstag alles Gute.

Karl-Josef Prommersberger

Bad Neustadt/Saale im November 2015 